\title{
LESÕES ASSOCIADAS À LAMINITE SUBCLÍNICA E EXAME RADIOGRÁFICO DIGITAL EM VACAS DA RAÇA HOLANDÊS NO PERÍODO TRANSICIONAL
}

\section{LESIONS ASSOCIATED WITH SUBCLINICAL LAMINITIS AND DIGITAL RADIOGRAPHIC EXAM IN HOLSTEIN COWS IN THE TRANSITIONAL PERIOD}

\author{
Antonio Amaral Barbosa ${ }^{*}$ \\ Charles Martins Ferreira1 \\ Márcio Nunes Corrêa ${ }^{1}$ \\ Elizabeth Schwegler ${ }^{2}$ \\ Raquel Fraga e Silva Raimondo 3 \\ Francisco Augusto Burkert Del Pino ${ }^{1}$ \\ ${ }^{1}$ Universidade Federal de Pelotas, Pelotas, RS, Brasil \\ ${ }^{2}$ Universidade Federal do Pampa, Uruguaiana, RS, Brasil \\ ${ }^{3}$ Universidade Federal do Rio Grande do Sul, Porto Alegre, RS, Brasil \\ *Autor para correspondência - antoniobarbosa.vet@hotmail.com
}

\section{Resumo}

A laminite subclínica é responsável por grandes prejuízos na pecuária leiteira, sendo o seu diagnóstico de grande relevância para evitar reflexos negativos na produção. Portanto, o objetivo deste trabalho foi relacionar lesões associadas à laminite subclínica no período de transição de vacas da raça Holandês com a ocorrência ou não de sinais radiográficos vinculados a essa enfermidade. As vacas foram avaliadas durante o período de transição e divididas em dois grupos (com e sem sinais de laminite subclínica) de acordo com as lesões associadas a essa enfermidade subclínica. A dinâmica radiológica foi realizada em dois momentos durante o período de transição, com o propósito de determinar possíveis alterações de posicionamento capsular e degeneração óssea da terceira falange (F3). Não foram observados efeitos da presença de lesões associadas à laminite subclínica frente ao posicionamento da terceira falange à cápsula do casco $(\mathrm{p}>0,05)$. Diferenças radiográficas significativas $(\mathrm{p}<0,05)$ angulares entre a F3 e a cápsula do casco foram observadas entre as unhas lateral e medial do mesmo dígito, mas não entre grupos ( $\mathrm{p}>0,05)$. Assim, neste estudo, vacas leiteiras no período de transição com lesões associadas à laminite subclínica não apresentaram sinais radiográficos de desalinhamento falangeano ao estojo córneo digital.

Palavras-chave: casco; bovinos; exames complementares; pododermatite asséptica.

\begin{abstract}
Subclinical laminitis has been responsible for economic losses in dairy industry; thus, its diagnostic is very important to prevent negative responses to milk production industry. The aim of this study was to identify lesions associated with subclinical laminitis during the transition period with presence or absence of radiographic signs linked to this disease in Holstein cows. The cows were evaluated during the transition period and categorized into two groups (with and without laminitis) according to the lesions associated with this subclinical disease. The radiological dynamic was performed in two target points during the transition period (prepartum and postpartum), with the objective of determining capsular position possible alterations and third phalanx bone degeneration (F3). There was no effect of the presence of lesions associated with subclinical laminitis regarding the position of the third phalanx to the hoof wall $(p>0.05)$. Significant radiographic angular differences $(p<0.05)$ between F3 and the
\end{abstract}


hoof wall were observed between the lateral and medial nails of the same digit, but not within groups $(p>0.05)$. Overall, this study did not show radiographic signs of phalangeal misalignment in the hoof of dairy cows with lesions associated with subclinical laminitis during the transition period .

Keywords: aseptic pododermatitis; bovines; complementary exams; hoof.

Enviado em: 21 janeiro de 2015

Aceito em 10 maio 2016

\section{Introdução}

Diferentes fatores estão relacionados com o aumento da incidência de claudicações, especialmente aqueles relacionados à adoção de tecnologias de produção intensiva com o objetivo de maximizar a produção dos animais. Dentre essas tecnologias está a adoção de sistemas de estabulação permanente com pisos impermeabilizados ${ }^{(1)}$, o aumento da densidade animal e o manejo nutricional ${ }^{(2,3)}$.

A seleção genética tem sido feita unifatorialmente, com enfoque maior em produção leiteira, dando-se pouca ênfase na estrutura músculo esquelética desses animais. No contexto atual, os animais sofrem as consequências de desequilíbrios metabólicos, observados principalmente no período transicional, quando a vaca está sob maior desafio, tornando-se mais vulnerável a danos sistêmicos, muitas vezes irreversíveis, ao sistema locomotor ${ }^{(4)}$.

Dentre os problemas podais, cerca de $90 \%$ está relacionado ao casco, sendo $70 \%$ destes provocados por pododermatite asséptica difusa (laminite) ${ }^{(2,5)}$. Alguns trabalhos vêm demonstrando o reflexo negativo para a produção leiteira de animais acometidos por essa enfermidade, que pode se manifestar tanto de forma aguda, crônica ou subclínica ${ }^{(6)}$.

Algumas teorias buscam explicar os eventos fisiopatológicos vinculados a esta afecção digital, destacando-se as teorias enzimática, metabólica ${ }^{(7)}$, biomecânica, inflamatória e vascular ${ }^{(8)}$. Contudo, até o momento, são escassos os estudos científicos em bovinos com laminite que enfatizam consequências relacionadas às alterações radiográficas proporcionadas por este estágio da enfermidade durante o período de transição.

Independente da teoria, aparecem as mesmas lesões associadas à enfermidade, porém é possível que ocorram diferenças quanto à severidade dessas lesões. Os animais que se enquadram dentro da categoria subclínica apresentam lesões macroscópicas digitais associadas. Dentre elas destacam-se principalmente: hemorragia de sola, úlcera de sola, dupla sola ${ }^{(9,10)}$, doença da linha branca ${ }^{(9)}$, hemorragia e separação da linha branca e deformação da unha ${ }^{(10)}$.

Os métodos diagnósticos frequentemente utilizados baseiam-se principalmente em observações de escore de locomoção e sinais clínicos específicos do sistema podal. A utilização de métodos complementares de diagnóstico em bovinos, como o diagnóstico por imagem radiológica, não é rotina na pecuária leiteira, diferentemente da crucial importância que é dada a esse método na avaliação de equinos. Com isso, muitas informações importantes do sistema ortopédico digital, associadas a essa enfermidade, deixam de ser observadas.

Contudo, até o momento são escassos os estudos sobre as diferentes formas de apresentação da laminite, sendo que ainda existem divergências no que diz respeito aos mecanismos da fisiopatologia da doença propostos para equinos ${ }^{(11)}$. Portanto, o objetivo deste estudo foi verificar lesões associadas à laminite subclínica com a ocorrência de sinais radiográficos a essa enfermidade durante o período de transição.

\section{Materiais e Métodos}

O experimento foi realizado em uma propriedade leiteira comercial localizada no sul do Brasil. Foram utilizadas 17 vacas, multíparas da raça Holandês, com três lactações, produção média de 30,67士5,39 
litros de leite aos 70 dias em lactação (DEL) e peso vivo de $656,89 \pm 92,6 \mathrm{Kg}$. Os animais foram monitorados de 14 dias antes do parto até 70 dias após o parto. As vacas foram ordenhadas duas vezes ao dia com intervalo de 12 horas e submetidas ao mesmo manejo semi-extensivo, recebendo dieta concentrada, como consta na Tabela 1 , segundo $\mathrm{NRC}^{(12)}$.

Tabela 1. Ingredientes disponíveis diariamente para as vacas leiteiras na dieta $(\mathrm{kg})$ e sua composição no pré e pós-parto

\begin{tabular}{llllll}
\hline Alimentos & \multicolumn{4}{l}{ Pré-parto (\%) } & \multicolumn{2}{l}{ Pós-Parto (\%) } \\
\hline \multirow{2}{*}{ Matéria Seca } & Forragem & Concentrado & Forragem & Pré-secado & Concentrado \\
FDN & 89,2 & 87,67 & 27,4 & 52,94 & 87,31 \\
FDA & 67,65 & 47,42 & 64,32 & 63,46 & 32,57 \\
Proteína total & 51,37 & 13,56 & 41,74 & 45,75 & 13,14 \\
Extrato Etéreo & 9,16 & 15,61 & 9,84 & 8,88 & 14,92 \\
Minerais totais & 1,73 & 3,57 & 2,02 & 2,00 & 4,01 \\
\hline
\end{tabular}

A metodologia empregada para o diagnóstico de laminite subclínica foi a utilizada por Falcão ${ }^{(13)}$. O grupo laminite subclínica (VCL) foi categorizado pela presença de duas ou mais lesões associadas a essa enfermidade no dígito e o grupo $(\mathrm{n}=12)$ sem lesões associadas à laminite (VSL) foi composto por animais com ausência ou presença de no máximo uma lesão associada $(n=5)$, como descrito no Quadro 1.

Todas as fêmeas bovinas que fizeram parte do grupo experimental foram submetidas à avaliação dinâmica de locomoção (escore de locomoção) e não apresentaram nenhum grau de instabilidade ortopédica.

Quadro 1. Lesões macroscópicas identificadas no dígito das fêmeas bovinas associadas à condição subclínica de laminite consideradas para a classificação dos grupos experimentais $^{(2,3,14-16)}$

\begin{tabular}{|l|l|}
\hline 01- Hemorragia de sola & 10 - Abscesso de sola \\
\hline 02- Hemorragia na linha branca & 11 - Abscesso de talão \\
\hline 03- Hemorragia na muralha & 12 - Abscesso de linha branca \\
\hline 04- Hemorragia no talão & 13 - Sola macia e amarelada \\
\hline 05- Úlceras de sola & $14-$ Sola dupla \\
\hline 06- Úlcera de pinça & 15 - Fenda na muralha \\
\hline 07- Alargamento da linha branca & 16 - Casco em tesoura \\
\hline 08- Fissuras na linha branca & $17-$ Casco em saca rolha \\
\hline 09- Doença da linha branca & 18 - Linhas de estresse na muralha \\
\hline
\end{tabular}


A avaliação radiográfica podal foi realizada com aparelho de radiografia digital (Cuattro ${ }^{\circledR}$ modelo Slate 2; 68Kv; 0,05 seg;) nos membros torácicos, bilateralmente, em projeção látero-medial com afastamento entre as unhas, no intuito de evitar sobreposição (Figura 1). A dinâmica radiológica foi realizada 14 dias antes da data prevista do parto e nos 70 dias após o mesmo, com o propósito de determinar possíveis alterações angulares ${ }^{(17)}$ e degeneração óssea da terceira falange (F3).
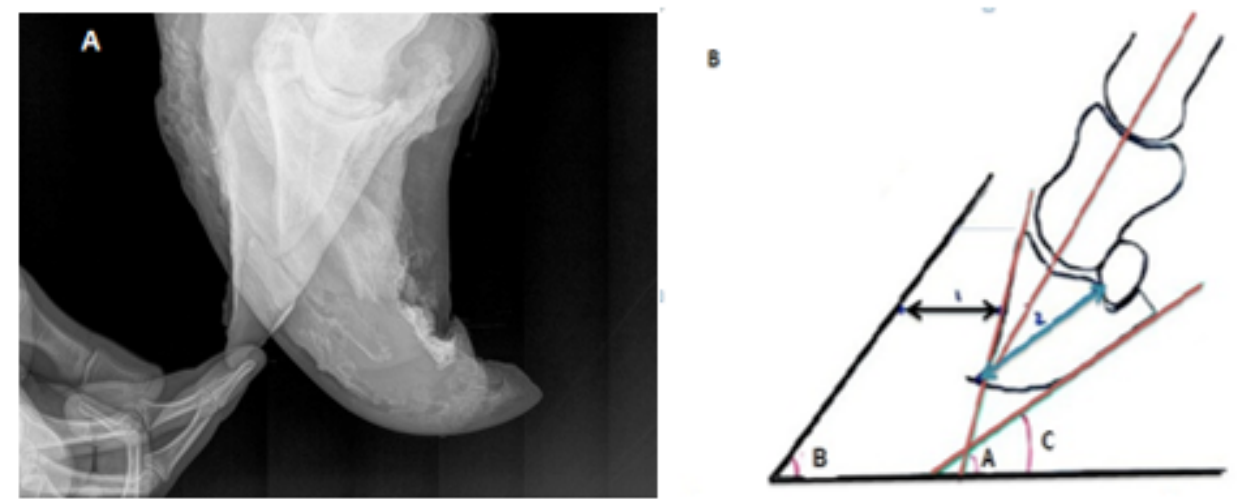

Figura 1. Método de posicionamento radiográfico dos dígitos para obtenção das imagens radiológicas (A). Mensuração da angulação da F3 dos bovinos frente ao seu posicionamento ao estojo córneo $(\mathrm{B})^{(17,18)}$. Ângulos: A: ângulo entre a face dorsal da terceira falange (F3) e o chão; B: ângulo entre a face dorsal do casco e o chão; C: ângulo entre a face dorsal da F3 e o chão; 1: espessura da parece; 2: longitude palmar da F3.

Para as análises dos dados, foi utilizada análise de variância simples (ANOVA One Way AoC), considerando-se os efeitos da presença ou não de sinais de laminite nos diferentes grupos frente ao posicionamento da terceira falange à cápsula do casco e diferenças de sinais radiográficos entre dígitos e unhas (Statistix8.0, 2013).

\section{Resultados e Discussão}

Dentre as lesões observadas pode-se verificar que a reação óssea proliferativa na região no processo extensor foi a mais expressiva $(n=68)$ nos animais, seguida dessa mesma lesão com extensão à articulação interfalangeana distal e fraturas. Porém, é importante ressaltar que nenhum animal possuía ambos os membros torácicos hígidos.

Efeitos traumáticos biomecânicos ao processo extensor representaram uma condição crônica observada em $100 \%$ dos animais e que não pode ser desvinculada de afecções clínicas prévias como erosão de talão ${ }^{(9)}$ e/ou úlcera de sola (lesões comuns em vacas que sofrem de laminite). Tais enfermidades proporcionam dor aguda palmar ao dígito, alterando a biomecânica de apoio por meio da deformidade flexural interfalangeana imposta pelo quadro álgico agudo. Apesar de ser uma alteração esperada em equinos, o desalinhamento falangeano com a cápsula do casco (rotação de F3) não foi uma condição encontrada em bovinos, embora os mesmos detenham lesões na região do processo extensor, a movimentação falangeana não foi constatada.

O método de avaliação radiográfica utilizado por Belotta et al. ${ }^{(19)} \mathrm{em}$ seu estudo retrospectivo com animais com desordens locomotoras diagnosticou a rotação de falange como uma das enfermidades 
mais recorrentes em equinos. Este fato não foi observado neste estudo nas vacas com mais de duas lesões associadas à laminite subclínica $(\mathrm{p}>0,05)$ (Tabela 2). Isto caracteriza que esses sinais digitais macroscópicos vinculados à condição de enfermidade laminar subclínica não foram associados ao desequilíbrio de posicionamento falangeano à cápsula do casco. Sendo assim, reduziram-se as possibilidades de efeitos biomecânicos dolorosos sobre a dinâmica de apoio do dígito no solo, que de certa forma pôde ser comprovado pela ausência de claudicação das vacas que apresentaram lesões associadas à laminite.

Apesar dos sinais evidenciados, uma das hipóteses que explicaria a ausência de sinais álgicos no sistema ortopédico está vinculada com a anatomia e a biomecânica de movimento no dígito em bovinos, possuindo caráter relevante a ser considerado no grau de manifestação dessa enfermidade. Acredita-se que o casco biungulado nesses animais pode amenizar a intensidade dos sinais clínicos, uma vez que possuem o amortecimento podal compartilhado entre as lâminas, os coxins e a sola, ${ }^{(20)}$ distribuindo a amortização e proporcionando menor movimentação falangeana dentro do aparelho suspensório do casco. Isso difere do que alguns estudos sobre equinos vêm enfatizando. Essa espécie é ungulada e possui sua biomecânica de apoio do dígito no solo amortecida somente pelas lâminas epidérmicas, o que contribui para a maior severidade e prevalência de afecções podais ${ }^{(8)}$.

Tabela 2. Mensuração radiográfica da F3 frente ao seu posicionamento ao estojo córneo do casco (média $(\mathrm{X}) \pm$ erro padrão $(\mathrm{SE})$ em vacas com e sem lesões associadas à laminite subclínica

\begin{tabular}{|c|c|c|c|c|c|c|}
\hline & $\mathrm{N}$ unhas & $\mathrm{A}$ & $\mathrm{B}$ & $\mathrm{C}$ & 1 & 2 \\
\hline $\mathrm{VCL}^{1}$ & 48 & $46,9(0,4)$ & $50,1(0,5)$ & $13,1(0,3)$ & $14,5(0,3)$ & $60,2(0,8)$ \\
\hline $\mathrm{VSL}^{2}$ & 20 & $46,3(0,7)$ & $49,8(0,8)$ & $12,2(0,6)$ & $15,1(0,4)$ & $57,8(1,3)$ \\
\hline Total & 68 & & & & & \\
\hline
\end{tabular}

Diferenças radiográficas significativas ( $p>0,05)$ angulares $(A, B, C, 1$ e 2) entre a F3 e a cápsula do casco foram observadas entre as unhas lateral e medial do mesmo membro, mas não entre grupos de vacas com e sem lesões associadas à laminite (Tabela 3). Em outras palavras, existe diferença somente entre a unha lateral e medial do mesmo animal, mas não entre animais. Isso caracteriza uma condição anatômica específica à unha e não à enfermidade, de acordo com o que descrevem Nicolleti $^{(2)}$ e Van Der Tol et al. ${ }^{(21)}$.

A diferença angular da F3 frente ao estojo córneo entre as unhas pode estar relacionada ao desequilíbrio látero-medial digital anterior promovido pelo centro gravitacional localizado exatamente sob o apêndice xifoide, caracterizando maior pressão biomecânica à locomoção sob a unha medial ${ }^{(2,21)}$.

Lesões radiográficas vinculadas ao osso terceira falange como osteólise, reação óssea proliferativa do processo extensor associada ou não a fraturas desta região anatômica e ósteo artrite interfalangeana distal foram observadas em ambos os grupos $(\mathrm{p}>0,05)$. Esses fatos não estão ligados ao momento subclínico, mas podem estar relacionados a uma condição dolorosa de laminite aguda prévia, pois as lesões associadas à laminite subclínica sugerem isso.

Baseado na ausência de diferenças angulares na posição da F3 frente ao estojo córneo, acreditase que a presença de lesões associadas à laminite encontradas nos cascos desses bovinos seja, provavelmente, devido a desequilíbrios sistêmicos e/ou metabólicos antecedentes, eventualmente não identificados clinicamente, expressos visualmente pelas lesões mensuradas neste estudo, traduzidas, não obrigatoriamente, como uma condição subclínica momentânea. 
Tabela 3. Mensuração radiográfica da $\mathrm{F} 3$ dos bovinos frente ao seu posicionamento ao estojo córneo do casco em vacas com e sem laminite subclínica

\begin{tabular}{|c|c|c|c|c|c|c|c|c|c|c|c|}
\hline & \multirow[t]{2}{*}{$\mathbf{N}$} & \multicolumn{2}{|c|}{$\mathbf{A}$} & \multicolumn{2}{|c|}{ B } & \multicolumn{2}{|c|}{ C } & \multicolumn{2}{|c|}{1} & \multicolumn{2}{|c|}{2} \\
\hline & & Med. & Lat. & Med. & Lat. & Med. & Lat. & Med. & Lat. & Med. & Lat. \\
\hline $\mathrm{VCL}^{1}$ & & $45,7^{\mathrm{b}}$ & $48,2^{a}$ & 49,3 & 50,8 & $11,7^{b}$ & $14,6^{2}$ & $15,8 \approx$ & $13,2^{\mathrm{b}}$ & $63,6^{a}$ & $56,7^{b}$ \\
\hline $\mathbf{P}$ & 48 & 0,01 & & $\mathrm{Ns}$ & & 0,01 & & 0,01 & & 0,01 & \\
\hline $\mathrm{VSL}^{2}$ & & $44,9^{b}$ & $47,8^{a}$ & 49 & 50,6 & $10,2^{b}$ & $14,2^{\mathrm{a}}$ & $16,2^{\text {a }}$ & $13,9^{b}$ & 59,9 & 55,8 \\
\hline $\mathbf{P}$ & 20 & 0,05 & & $\mathrm{Ns}$ & & 0,01 & & 0,01 & & 0,06 & \\
\hline
\end{tabular}

\section{Conclusões}

Vacas leiteiras no período de transição com lesões associadas à laminite subclínica não apresentaram sinais radiográficos de alterações angulares entre a terceira falange e o estojo córneo. As lesões crônicas na região do processo extensor podem ser uma consequência clínica em vacas com episódios de laminite, sem rotação de falange.

\section{Agradecimentos}

Os autores agradecem à Coordenadoria de Aperfeiçoamento de Pessoal de Nível Superior (CAPES) à Fundação de Amparo à Pesquisa do Estado do Rio Grande do Sul (FAPERGS), ao Conselho Nacional de Desenvolvimento Científico e Tecnológico (CNPQ) pelo apoio financeiro e à Granja 4 irmãos pela disponibilidade da execução do projeto.

\section{Referências}

1. Silva MAF. Podologia em bovinos: conceitos basilares. Vila Real: UTAD; 2009. 64 p.

2. Nicoletti JLM. Manual de podologia bovina. Barueri: Manole; 2004. 126p. Portuguese.

3. Ferreira PM. Enfermidades podais em rebanho leiteiro confinado. 2003. 79f. Tese (Doutorado em Ciência Animal) - Escola de Veterinária, Universidade Federal de Minas Gerais, Belo Horizonte.

4. Van der Waaij EH, Holzhauer M, Ellen E, Kamphuis C, and de Jong, G. Genetic parameters for claw disorders in dutch dairy cattle and correlations with conformation traits. J Dairy Sci. 2005; 88 (10):3672-8.

5 Silva LAF, Silva LM, Romani AF, Rabelo RE, Fioravanti MCS, Souza TM, Silva CA. Características clínicas e epidemiológicas das enfermidades podais em vacas lactantes do município de Orizona - GO. [Clinical and epidemiological characteristics of foot diseases in dairy cows in the municipality of Orizona - GO]. Ciência Animal Brasileira [Internet]. $2001 \mathrm{Jul} / \mathrm{Dec}$ [cited 2014 Aug 21]; 2(2):119-126. Available from: http://www. 
revistas.ufg.br/index.php/vet/article/view/264/236. Portuguese.

6. Warnick LD, Janssen D, Guard CL. The effect of lameness on milk production in dairy cows. Journal of Dairy of Science [Internet]. 2001 Sep [cited 2014 Jul 18]; 84(9):1988-1997. Available from: http://www.ncbi. nlm.nih.gov/pubmed/11573778. English.

7. Mulligan FJ, Doherty ML. production diseases of the transition cow. J Vet. 2008; 176 (1): 3-9.

8. Orsini JA, Parsons CS, Capewell L, Smith G. Prognostic indicators of poor out come in horses with laminitis at a tertiary care hospital. The Canadian Veterinary Journal [Internet]. 2010 [cited 2014, Aug 21]; 51(6):623628.Available from: http://www.ncbi.nlm.nih.gov/pmc/articles/ PMC2871359/pdf/cvj_06_623.pdf. English.

9. Flor E, Tadich N. Claudicaciones en vacas de rebaños lecheros grandes y pequeños Del sur de Chile. Archivos de medicina veterinária [Internet]. 2008 [cited 2014 Aug 22]; 40(2):125-134. Available from: http:// www.scielo.cl/pdf/amv/v40n2/art03.pdf. Spanish.

10. Ossent P, Lisher CJ. Bovine laminitis: the lesions and their pathogenesis. Practice; 1998. p. 415-427. English.

11. Pollitt CC, Kyaw-Tanner M, French KR, Van Eps AW, Hendrikz JK, Daradka M. equine laminitis current concepts. Publication No. 08/062, Project No. RIRDC UQ-118A. Queensland - Australia. 2008; p.27,42.12.

12. NATIONAL RESEARCH COUNCIL - NRC. Nutrients requirements of the dairy cattle. 7 ed. Washington, D.C.: 2001. 381p. English.

13. Falcão, H. M. Lesões na junção derme-epiderme do casco de vacas de aptidão leiteira de descarte com e sem sinais clínicos de laminite. Dissertação (Mestrado em Medicina e Cirurgia Veterinárias) - Escola de Veterinária, Universidade Federal de Minas Gerais, Belo Horizonte. 2010. [citado 2014 Ago 24]; 74f. Disponível em: http://hdl.handle.net/1843/BUOS-8SDM87. Portuguese.

14. Nocek JE. Bovine acidosis: implications in laminitis. Journal of Dairy Science [Internet]. 1997 [cited 2014 Aug 21]; 80(5):1005-1028. Available from: http://www.journalofdairyscience.org/article/S00220302(97)76026-0/pdf. English.

15. Andreson DE, Rings M. Current Veterinary Therapy: Food Animal Practice. 5st ed. St. Louis: Saunders Elsevier; 2008. 736p. English.

16. Greenough PR. The laminitis syndrome. In: Ibid. (Ed.), Bovine Laminitis and Lameness: a hands-on approach. Toronto: Elsevier; 2007. p. 36-54. English

17. Thrall DE. Text book of veterinary diagnostic radiology. 4ed. Philadelphia: W.B. Sauders; 2002. 429p. English.

18. García JS, Pérez ECP. Sindrome infosura. In: I Curso de Podiatria Equina Especializada da Universidade de Évora. Évora: Unievora; 2007. 27-30p. Portuguese.

19. Belotta AF, Velasquez DRB, Carneiro JAM, Bernardo JO, Nitta TY, Araújo CET et al. radiográficos das afecções do aparelho locomotor de equinos: estudo retrospectivo de 1480 casos (2000 a 2012). J Vet. e Zootec. 2014; 21 (4): 634-645.

20. König HE, Liebich H. Anatomia dos animais domésticos: texto e atlas colorido. Porto Alegre: Artmed; 2004. 787p. Portuguese.

21. Van Der Tol PPJ, Metz JHM, Noordhuizen-Stassen EN, Back W, Braam CR, Weijs WA. The pressure distribution under the bovine claw during square standing on a flat substrate.Journal of Dairy Science [Internet]. 2002 Jun [cited $2014 \mathrm{Jul}$ 20]; 85(6):1476-1481. Available from: http://www.journalofdairyscience.org/article/ S0022-0302(02)74216-1/pdf. English. 